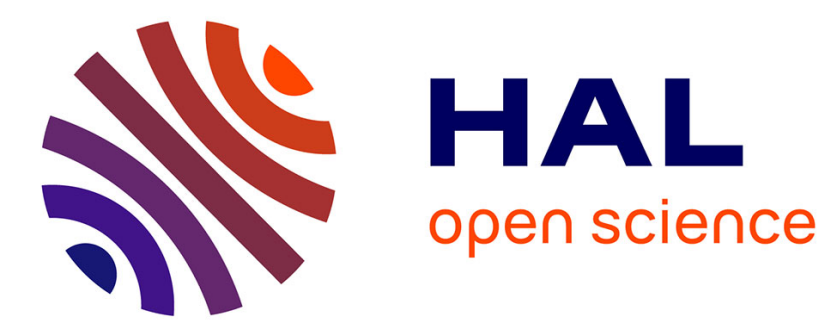

\title{
Performance analysis of the on-the-air activation in LoRaWAN
}

Joël Toussaint, Nancy El Rachkidy, Alexandre Guitton

\section{To cite this version:}

Joël Toussaint, Nancy El Rachkidy, Alexandre Guitton. Performance analysis of the on-the-air activation in LoRaWAN. IEEE 7th Annual Information Technology, Electronics and Mobile Communication Conference (IEMCON), Oct 2016, Vancouver, Canada. pp.1 - 7, 10.1109/IEMCON.2016.7746082 . hal-01471659

\section{HAL Id: hal-01471659 \\ https://hal.uca.fr/hal-01471659}

Submitted on 20 Feb 2017

HAL is a multi-disciplinary open access archive for the deposit and dissemination of scientific research documents, whether they are published or not. The documents may come from teaching and research institutions in France or abroad, or from public or private research centers.
L'archive ouverte pluridisciplinaire HAL, est destinée au dépôt et à la diffusion de documents scientifiques de niveau recherche, publiés ou non, émanant des établissements d'enseignement et de recherche français ou étrangers, des laboratoires publics ou privés. 


\title{
Performance analysis of the on-the-air activation in LoRaWAN
}

\author{
Joël Toussaint ${ }^{(1,2)}$, Nancy El Rachkidy ${ }^{(3,2)}$, Alexandre Guitton ${ }^{(3,2)}$ \\ (1) Clermont Université, Université d'Auvergne, BP 10448, F-63000 Clermont-Ferrand, France \\ (2) CNRS, UMR 6158, LIMOS, F-63175 Aubière, France \\ (3) Clermont Université, Université Blaise Pascal, BP 10448, F-63000 Clermont-Ferrand, France \\ Emails: joel.toussaint@udamail.fr, \{nancy.el_rachkidy,alexandre.guitton\}@univ-bpclermont.fr
}

\begin{abstract}
Long-range low-power communications are starting to replace short-range low-power communications in monitoring applications based on wireless sensor networks, both in rural and urban environments, due to the small deployment cost of long-range technologies. In this paper, we focus on the MAC layer of the recent LoRaWAN (long-range wide area network) standard, and specifically on the on-the-air activation procedure, which defines how nodes join an existing network. We propose a Markov chain model of the on-the-air activation, and we derive the expected delay and the expected energy required to join the network. We analyze the impact of several parameters on these metrics, including traffic conditions, duty cycles, and channel availability. We also discuss the impact of the regional settings of the standard. To the best of our knowledge, this is the first analysis of the MAC layer of LoRaWAN.
\end{abstract}

\section{INTRODUCTION}

Wireless sensor networks (WSNs) are largely used to monitor rural environments [1], [2] and urban environments [3]. Indeed, WSNs have a strong potential thanks to their low cost, their autonomy in energy, and the disposability of the individual sensors. Wireless sensor networks have also found many applications with the Internet of Things paradigm, where the Internet is coupled with the physical world through sensors and actuators. The IEEE 802.15.4 standard [4], initially designed in 2003 [5], is by far the main standard for wireless communications in WSNs [1], [6].

Several researchers have analyzed the performance of the MAC layer of IEEE 802.15.4, as it has a direct impact on the applications using this standard. Most of the analytical analyses propose a Markov chain model, adapted to different cases. For instance, the behavior of a node during the contention access period of the beacon-enabled mode is studied in [7] when the network is saturated, and in [8] when the network is not saturated. In [9], the authors study the traffic differentiation during the contention access period. In [10], the authors model the impact of a lossy channel (due to interference and poor link quality) on the communication. In [11], the authors model the accumulation of the traffic due to the long inactive periods of the beacon-enabled mode.

IEEE 802.15.4 has only short-range capabilities: the communication range traditionally varies between tens of meters (in indoor environment) to up to one hundred meters (in outdoor environment and favorable conditions, such as line of sight). To compensate for this limitation, it is possible to deploy more nodes in order to achieve network connectivity. This comes at an extra cost, which might be prohibitive in large-scale applications such as volcano monitoring, forest monitoring, or large urban deployments.

Recently, long-range low-power wireless standards have emerged, such as Sigfox [12], Ingenu [13] and LoRaWAN [14]. These standards enable one-hop communications ranging from $2 \mathrm{~km}$ in dense urban environments to $30 \mathrm{~km}$ in rural environments. These standards bring high expectations, as they create new possibilities for low-power, largescale monitoring applications. Since most of these standards are proprietary, few research works have focused on them. One of these works is [15], in which the authors have studied the physical layer of LoRaWAN in a real deployment.

In this paper, we study the MAC layer of the LoRaWAN standard (which is open and freely available), and we focus on the on-the-air activation procedure, which allows nodes to join an existing network. To do this, we propose a Markov chain model of the activation procedure, and we derive two important performance metrics: the expected delay to complete the activation, and the required energy. The study is based on the EU863-870 MHz regional setting (also other regional settings are discussed in the paper) for a single gateway. To the best of our knowledge, this is the first analytical study of the MAC layer of LoRaWAN.

The remainder of this paper is organized as follows. Section II describes the LoRaWAN standard and focuses on the activation procedure. Section III proposes our Markov chain model. Section IV presents our performance evaluation for different parameters. Section V initiates a discussion, which includes the impact of the other regional settings of LoRaWAN. Finally, Section VI concludes our work.

\section{DESCRIPTION OF THE LORAWAN STANDARD}

LoRaWAN [14] is a LPWAN (Low-Power Wide Area Network) communication standard. It aims to exchange data (such as alarms, meters, or monitoring devices) between low-power devices and a network server, through gateways over long distances. LoRaWAN achieves long-range radio communications at small bit rates (from 0.3 to $50 \mathrm{kbps}$ ) and is therefore well suited for many applications, including the Internet of Things. Experiments show transmission ranges of up to $1.3 \mathrm{~km}$ in an 
urban environment with a frame loss of $10 \%$ [16], [15], and of up to $21.6 \mathrm{~km}$ in line of sight conditions without loss [17].

LoRaWAN architecture is based on a star topology. Enddevices exchange frames with gateways. Gateways relay the frames to the network server thanks to a classical internet infrastructure. Long-range radio links are achieved with a proprietary modulation technique called Lora ${ }^{\mathrm{TM}}$, developed by the Semtech company, and based on the Chirp Spread Spectrum (CSS) technology ${ }^{1}$. LoRa uses ISM bands, depending on regional settings: we focus mainly on the European 863$870 \mathrm{MHz}$ band. LoRaWAN also proposes an Adaptive Data Rate (ADR) mechanism in order to adapt the transmission characteristics (data rate, spreading factor, and bandwidth) to the propagation conditions. Data rates range from DR0 (the lowest data rate) to DR7 (the highest data rate).

LoRaWAN defines three classes of end-devices: class A, class B and class C. Class A is the default class, and is mandatory for all devices. Communication is initiated by end-devices: uplink communications (that is, to the gateway) occur when the end-device has data to transmit, and downlink communication (that is, from the gateway) are possible only after an uplink transmission. Uplink communications are performed on a random channel. A long preamble is sent before the payload. Downlink transmissions have to start at the beginning of two receive windows. Downlink communications during the first receive window are performed on the same channel as the uplink transmission, with the same data rate (by default). Downlink communications during the second receive window are performed on a fixed channel and with a fixed data rate. After having sent a frame on a given channel, the node refrains from using any channel of the sub-band during $t_{\text {off }}=t_{\text {air }} / \delta-t_{\text {air }}$ time units, where $t_{\text {air }}$ is the time on air of the frame sent, and $\delta$ is the duty-cycle for this sub-band (which is limited to $1 \%$ ). Class B introduces beacons which allow enddevices to be synchronized and enable scheduled downlink communications. Class $\mathrm{C}$ removes the energy constraint by allowing nodes to continuously listen to the medium.

An activation procedure is required for end-devices to participate to network activities. LoRaWAN defines two activation procedures: the activation by personalization (ABP), and the on-the-air activation (OTAA). In the ABP, end-devices possess in their memory the required information: thus, no communication is required to join the network. In the OTAA, the enddevice sends a join request ${ }^{2}$ to the gateway, which forwards the frame to the network server. The network server responds with a join accept ${ }^{3}$, which is forwarded by the gateway. The gateway can transmit this join accept during either the first receive window (which occur five seconds after the end of transmission of the join request) or during the second receive window (which occur six seconds after the end of transmission of the join request). In this paper, we focus on OTAA. Note that the activation procedure is the same for all three classes.

\footnotetext{
${ }^{1}$ Note that in Europe and China, the highest data rate available (50 kbps) is achieved using an FSK modulation.

${ }^{2}$ The size of the join request payload is 18 bytes, see 6.2 .4 of [14].

${ }^{3}$ The size of the join accept payload is 12 bytes, see 6.2 .5 of [14].
}

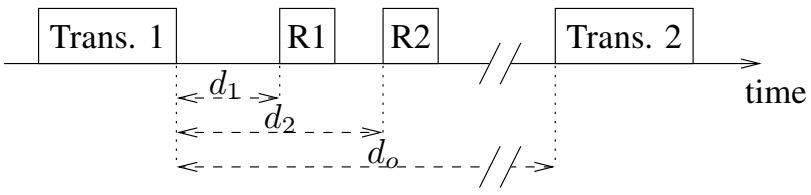

Figure 1. Medium access in the class A of LoRaWAN.

Figure 1 depicts the medium access in the class A of LoRaWAN, which is used during the activation procedure, and during the data exchange (with different timers). After a transmission, a first receive window is opened $\left(d_{1}=5\right.$ during the activation, and $d_{1}=1$ once the node is activated). If there is no preamble detected during the first receive window, or if a transmission for another node is detected, the second receive window is opened $\left(d_{2}=6\right.$ during the activation, and $d_{2}=2$ once the node is activated). Independently of the fact that a preamble is received or not, the channel (as well as other channels of the same sub-band) cannot be used for a duration $d_{o}$, depending on the duty-cycle.

\section{MARKOV CHAIN MODEL}

In this section, we start by presenting our hypotheses. Then, we present our Markov chain model for the on-theair activation. Finally, we describe the computation of our two performance metrics: the expected delay to complete the activation procedure, and the expected energy required.

\section{A. Hypotheses}

We describe our simplification hypotheses in the following.

a) Hypotheses concerning the physical layer: We assume that when a transmitter sends a preamble, the receiving node detects this preamble, thanks to the robust CSS modulation which is central in the physical layer design of LoRaWAN. We assume that when several transmitters send a preamble simultaneously, the receiving node detects that the preamble is incorrect. In other words, we ignore the capture effect. Moreover, we assume that collisions between uplink and downlink frames might happen.

b) Hypotheses concerning the gateway: We assume that there is a single gateway. We assume that the gateway is sending only join accept frames (that is, there is no confirmed data requiring acknowledgments), and that the gateway is not sending a frame while a join request frame is sent.

c) Hypotheses concerning the nodes: We assume that nodes use the data rate DR0 for the activation procedure (which is the smallest data rate, ensuring the strongest link quality) and the data transmission (of activated nodes). We assume that the MAC payload of data frames is between 13 bytes and 18 bytes (this assumption has a small impact on the Markov chain, and is discussed in Section V). We also assume that the detection of the preamble lasts for the whole duration of the preamble.

\section{B. Model}

Figure 2 represents our Markov chain model for the activation procedure of LoRaWAN. In the send request state, the 
node sends a join request and goes to the receive 1 state. In the receive 1 state, the node opens the first receive window. If a preamble is detected, the node goes to the preamble 1 state, then to the check 1 state if the preamble is correct, and then to the activated state if the join accept is successfully received. Otherwise, the node goes either to the receive 2 state or to the wait state, depending on the duration of the potential preamble and frame received. In the receive 2 state, the node opens the second receive window, and follows a process similar to the receive 1 state. In the wait state, the node waits for a given amount of time until it can reuse a sub-band, and goes back to the send request state. Finally, in the activated state, the node has successfully joined the network and is ready to send data.

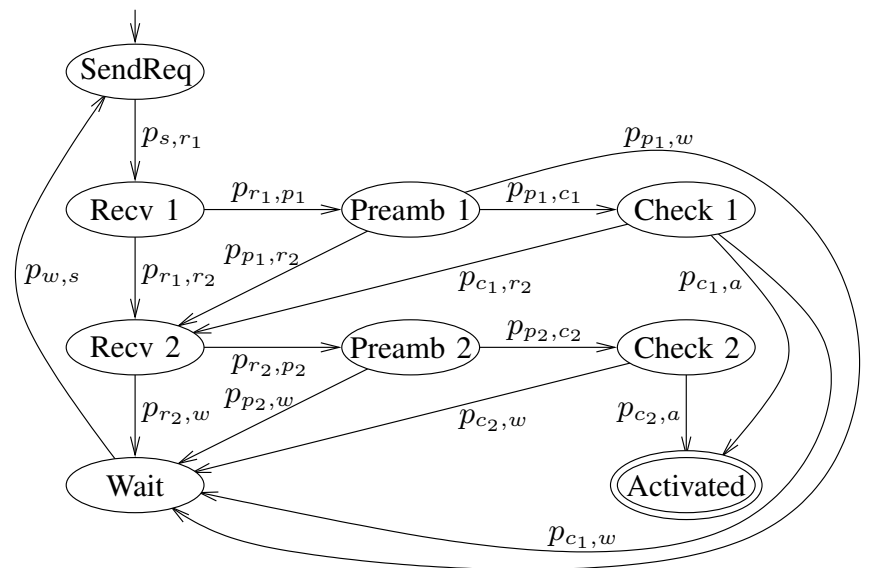

Figure 2. Markov chain model for the on-the-air activation of LoRaWAN.

We use the following notations. $n_{I}$ denotes the number of inactivated nodes (that is, nodes that are requesting to join the network), and $n_{A}$ the number of activated nodes (that is, nodes that have already joined the network). $n_{S B}$ is the number of sub-bands, and $n_{C}$ is the number of channels per sub-band. $\alpha \in[0 ; 1]$ represents the link quality: when $\alpha=1$, the frame error rate is $0 . \gamma$ determines whether the gateway sends the join accept during the first receive window $(\gamma=1)$ or during the second receive window $(\gamma=0) . \delta \in[0 ; 0.01]$ is the duty cycle of activated nodes per sub-band (which is limited to $1 \%$ per sub-band by the standard). Traffic is generated by activated nodes with probability $\tau_{A} \cdot \delta \cdot n_{S B}$, with $\tau_{A} \in[0 ; 1]$ : when $\tau_{A}=1$, the network is saturated.

Several probabilities of Figure 2 depend on the availability of a channel at a given time, which depends on the probability that no other node uses this channel. The probability that an inactivated node does not use a given channel is $q_{I}=1-\frac{0.001}{n_{C} \cdot n_{S B}}$. Indeed, $1-q_{I}$ is the probability that the node sends a join request on this channel (out of the $n_{C} \cdot n_{S B}$ possible channels), given the fact that the duty-cycle during the activation procedure is $0.1 \%$. The probability that an activated node does not use a given channel is $q_{A}=1-\frac{\delta \cdot \tau_{A}}{n_{C}}$. Indeed,

\footnotetext{
${ }^{4}$ According to paragraph 7.1 .2 of the standard, the duty-cycle limitation of join requests is $0.1 \%$, independently of the number of sub-bands (unlike the data duty-cycle limitation).
}

$1-q_{A}$ is the probability that the node sends a data frame on this channel (as it produces $\delta \cdot \tau_{A} \cdot n_{S B}$ data frames per time unit, distributed among $n_{S B}$ sub-bands and $n_{C}$ channels per sub-band).

Probability $p_{s, r_{1}}$ to open the first receive window after having sent a join request (see Equation 1) is equal to 1.

$$
p_{s, r_{1}}=1
$$

Probability $p_{r_{1}, r_{2}}$ is the probability that the node does not detect any preamble during the first receive window, and is given in Equation 2. It is equal to the probability that there is no transmission from the gateway, from any inactivated node, nor from any activated node. The first factor represents the absence of transmission from the gateway, and is obtained through the computation of the probability that the gateway is sending a join accept, which depends on the fact: (i) that there was no interference with the send request (modeled with $\left.q_{I}^{n_{I}} \cdot q_{A}^{n_{A}}\right)$, (ii) that the send request was successfully decoded (modeled with $\alpha$ ), and (iii) that the gateway replies during the first receive window (modeled with $\gamma$ ). The second factor represents the absence of transmission from inactivated nodes. The third factor represents the absence of transmission from activated nodes. Probability $p_{r_{1}, p_{1}}$ is obtained by complementarity (see Equation 3).

$$
\begin{aligned}
& p_{r_{1}, r_{2}}=\left(1-\alpha \cdot \gamma \cdot q_{I}^{n_{I}} \cdot q_{A}^{n_{A}}\right) \cdot q_{I}^{n_{I}} \cdot q_{A}^{n_{A}} \\
& p_{r_{1}, p_{1}}=1-p_{r_{1}, r_{2}}
\end{aligned}
$$

Probability $p_{p_{1}, w}$ is the probability that the preamble received during the first receive window is incorrect and that its duration exceeds the delay of $1 \mathrm{~s}$ between the two receive windows. Since the longest preamble duration is $401.41 \mathrm{~ms}$ (for DR0, which corresponds to a spreading factor of 12 and a bandwidth of $125 \mathrm{kHz}$ ), $p_{p_{1}, w}=0$ (see Equation 4). Probability $p_{p_{1}, c_{1}}$ is the probability that the preamble received is correct, and is given in Equation 5. It is equal to the probability that one preamble exactly is received given the fact that at least one preamble was sent. Let us denote by $x$ the number of preambles sent. $p_{p_{1}, c_{1}}=\mathcal{P}(x=1 \mid x \geq$ $1)=\mathcal{P}(x=1) / \mathcal{P}(x \geq 1)=\mathcal{P}(x=1) / p_{r_{1}, p_{1}} \cdot \mathcal{P}(x=1)$ is the sum of the following mutually exclusive probabilities: the probability that only the gateway sends a preamble, the probability that only one inactivated node sends a preamble, and the probability that only one activated node sends a preamble. Probability $p_{p_{1}, r_{2}}$ is obtained by complementarity (see Equation 6).

$$
\begin{aligned}
p_{p_{1}, w} & =0 \\
\mathcal{P}(x=1) & =\alpha \cdot \gamma \cdot q_{I}^{n_{I}} \cdot q_{A}^{n_{A}} \cdot q_{I}^{n_{I}} \cdot q_{A}^{n_{A}}+ \\
& \left(1-\alpha \cdot \gamma \cdot q_{I}^{n_{I}} \cdot q_{A}^{n_{A}}\right) \cdot q_{I}^{n_{I}-1}\left(1-q_{I}\right) n_{I} \cdot q_{A}^{n_{A}+} \\
& \left(1-\alpha \cdot \gamma \cdot q_{I}^{n_{I}} \cdot q_{A}^{n_{A}}\right) \cdot q_{I}^{n_{I}} \cdot q_{A}^{n_{A}-1}\left(1-q_{A}\right) n_{A} \\
p_{p_{1}, c_{1}} & =\mathcal{P}(x=1) / p_{r_{1}, p_{1}} \\
p_{p_{1}, r_{2}} & =1-p_{p_{1}, w}-p_{p_{1}, c_{1}}
\end{aligned}
$$

Probability $p_{c_{1}, a}$ is the probability that the received frame is the expected join accept frame, and is given in Equation 7. It 
is equal to the fact that the gateway received and decoded the join request, and that the join accept is successfully received and decoded. Probability $p_{c_{1}, r_{2}}$ is the probability that the received frame is not the correct join accept frame (otherwise, the transition would be $p_{c_{1}, a}$ ), and that it is small enough so that the reception duration does not exceed the delay between the two receive windows (otherwise, the transition would be $\left.p_{c_{1}, w}\right)$. It is given in Equation 8. Note that out of the three types of frames which can be received (join accept, join request, and data), only the join accept frames cause the node to open the second receive window. Indeed, the time on air of join accept frames (including the preamble) is $991.23 \mathrm{~ms}$ for DR0 (since the MAC payload length is 12 bytes), which means that the node has time to open the second receive window only when an incorrect join accept frame is received. The time on air of join request frames (including the preamble) and data frames of 13 bytes or more (including the preamble) is larger than $1 \mathrm{~s}$ for DR0, which means that the node does not have time to open the second receive window when such a frame is received. In this case, the node goes back directly to the wait state, which is modeled in Equation 9.

$$
\begin{aligned}
p_{c_{1}, a} & =\alpha \cdot \gamma \cdot q_{I}^{n_{I}} \cdot q_{A}^{n_{A}} \cdot q_{I}^{n_{I}} \cdot q_{A}^{n_{A}} \cdot \alpha \\
p_{c_{1}, r_{2}} & =\alpha \cdot \gamma \cdot q_{I}^{n_{I}} \cdot q_{A}^{n_{A}} \cdot q_{I}^{n_{I}} \cdot q_{A}^{n_{A}}(1-\alpha) \\
p_{c_{1}, w} & =1-p_{c_{1}, r_{2}}-p_{c_{1}, a}
\end{aligned}
$$

Probability $p_{r_{2}, p_{2}}$ is the probability that the node detects a preamble during the second receive window, and is given in Equation 10. Since the second receive window is opened on a channel that is exclusive to the gateway, it is not possible for other nodes to send on this channel ${ }^{5}$. Thus, $p_{r_{2}, p_{2}}$ only depends on the fact that the gateway received the join request correctly (modeled with $\alpha$ ) and without interference (modeled with $\left.q_{I}^{n_{I}} \cdot q_{A}^{n_{A}}\right)$, and that it replied on the second receive window (modeled with $1-\gamma$ ). Probability $p_{r_{2}, w}$ is obtained by complementarity (see Equation 11).

$$
\begin{aligned}
p_{r_{2}, p_{2}} & =\alpha(1-\gamma) q_{I}^{n_{I}} \cdot q_{A}^{n_{A}} \\
p_{r_{2}, w} & =1-p_{r_{2}, p_{2}}
\end{aligned}
$$

Probability $p_{p_{2}, c_{2}}$ is the probability that the node detects a preamble during the second receive window, and is given in Equation 12. Since only the gateway is allowed to transmit during the second receive window, it is not possible for the preamble to be received incorrectly. Probability $p_{p_{2}, w}$ is obtained by complementarity (see Equation 13).

$$
\begin{aligned}
p_{p_{2}, c_{2}} & =1 \\
p_{p_{2}, w} & =0
\end{aligned}
$$

Probability $p_{c_{2}, a}$ is the probability that the frame received during the second receive window is a join accept for the node, and is given in Equation 14. Since the gateway is the only node

\footnotetext{
${ }^{5}$ According to the standard for the EU863-870 MHz ISM band, the RX2 receive window is by default on channel $869.525 \mathrm{MHz}$ (see paragraph 7.1.7), and this channel is neither included in the default join request channel list (see Table 13 in paragraph 7.1.2) nor in the default data channel list (see Table 12 in paragraph 7.1.2)
}

allowed to send on the channel, and since we assumed that the gateway is always able to send a join accept, $p_{c_{2}, a}$ is equal to the probability that the join accept is successfully received by the node ${ }^{6}$. Probability $p_{c_{2}, w}$ is obtained by complementarity (see Equation 15)

$$
\begin{aligned}
p_{c_{2}, a} & =\alpha \\
p_{c_{2}, w} & =1-\alpha
\end{aligned}
$$

Probability $p_{w, s}$ to send a join request after the wait state (see Equation 16) is equal to one.

$$
p_{w, s}=1
$$

\section{Computation of performance metrics}

In the following, we use the Markov chain model to compute the expected delay and energy consumption of the activation procedure of LoRaWAN.

Our Markov chain is an absorbing chain, because it is possible to reach the activated state (which is absorbing) from any state. Let us denote by $N$ the fundamental matrix of our Markov chain: $N_{i, j}$ represents the expected number of visits of state $j$ when starting from state $i$. Since the initial state is the send request state, the expected number of visits $V$ can be computed by $V=1_{s} \cdot N$, where $1_{s}$ is a row vector having the first entry (representing the send request state) equal to 1 , and all other entries equal to 0 . In the remainder of this part, we compute the expected delay of the activation based on $V$ and on the delay of each state. We compute the expected energy of the activation procedure based on $V$, on the delay of each state, and on the energy of each state.

The expected delay of the activation procedure is equal to $V \cdot D$, where $D$ is a column vector having each entry representing the expected duration spent in the corresponding state. $D_{s}$ is the duration of the send request state, and is equal to the time between the beginning of the transmission of the send request and the start of the first receive window. Thus, $D_{s}$ is equal to the time on air of the join request plus five seconds. $D_{r_{1}}$ is at least the time required to detect a preamble (according to paragraph 3.3.3 of the standard): we assumed that $D_{r_{1}}$ is equal to the duration of a preamble. $D_{p_{1}}$ is equal to 0 , since the preamble 1 state is simply used to determine whether the received preamble was correct or not. $D_{c_{1}}$ has two terms: the first is the average time on air of received frames minus the duration of the preamble (which was already accounted for in $D_{r_{1}}$ ), and the second is the time before the second receive window. The first term can be computed using a weighted average, since the probability to receive a small join accept frame is $p_{c_{1}, a} / \alpha$, and since other frames (join requests or data frames of 13 to 18 bytes) have the same time on air duration. The second term is equal to the delay between the two receive windows minus the average time on air of the received frame. $D_{r_{2}}$ is equal to the duration of the preamble. $D_{p_{2}}$ is equal to $0 . D_{c_{2}}$ is the time on air of the join accept

\footnotetext{
${ }^{6}$ Note that it is not possible to receive a join accept intended to another inactivated node, as it would mean that the two join requests were sent simultaneously.
} 
frame minus the duration of the preamble. Finally, $D_{w}$ is the time during which the sub-band is unavailable after having sent a join request, divided by the number of available sub-bands.

The expected energy of the activation procedure is equal to $V \cdot E$, where $E$ is a column vector having each entry representing the expected energy consumption of the corresponding state. $E_{s}$ is the energy spent while transmitting the join request, plus the energy spent while waiting for the first receive window to open. $E_{r_{1}}$ (respectively $E_{r_{2}}$, and $E_{c_{2}}$ ) is the energy spent while listening to the potential preamble of the first receive window (respectively potential preamble of the second receive window, and frame of the second receive window). $E_{p_{1}}$ and $E_{p_{2}}$ are both equal to $0 . E_{c_{1}}$ has two terms: the first term is equal to the energy spent while receiving a frame (whose duration is given by the first term of $D_{c_{1}}$ ) and the second term is equal to the energy spent in idle mode before the second receive window opens (whose duration is the second term of $D_{c_{1}}$ ). Finally, $E_{w}$ is equal to the energy spent in idle mode while waiting for a channel to become available.

\section{Performance eValuation}

In this section, we evaluate the performance of the activation procedure, in terms of expected delay and expected energy consumption.

\section{A. Parameter settings}

We ran our simulations using Scilab, and we considered the following default parameters. The channel quality is high $(\alpha=0.99)$ and the network is saturated $\left(\tau_{A}=1\right)$. There are $n_{C}=3$ channels for each of the $n_{S B}=2$ sub-bands. There are $n_{I}=10$ inactivated nodes (in addition to the node being studied) and $n_{A}=10$ activated nodes. The first receive window is used by default $(\gamma=1)$, and the duty cycle per sub-band of activated nodes is $\delta=0.01$.

We used the energy consumption settings of the Semtech SX1272 component, designed for LoRaWAN: the transmit power is $90 \mathrm{~mA}$ (at $17 \mathrm{dBm}$ ), the reception power is 10.8 $\mathrm{mA}$, and the idle power is $0.1 \mathrm{~mA}$. The voltage is $1.5 \mathrm{~V}$.

From these settings, we obtained the following (rounded) values for vectors $D$ and $E$ (the order of states in the vectors being: send request, receive 1 , preamble 1 , check 1 , receive 2 , preamble 2, check 2, and wait):

$$
\begin{gathered}
D=\left(\begin{array}{llllllll}
6.16 & 0.40 & 0 & 0.60 & 0.40 & 0 & 0.59 & 576.96
\end{array}\right)^{T} \\
E=\left(\begin{array}{llllllll}
0.08 & 0.007 & 0 & 0.01 & 0.007 & 0 & 0.01 & 9.10^{-5}
\end{array}\right)^{T} .
\end{gathered}
$$

\section{B. Results}

Figure 3 presents the delay as a function of $\alpha$ and $\gamma$. The impact of the channel quality $\alpha$ on the expected delay is significant. When the channel quality is low $(\alpha=0.9)$, join requests or join accepts might be lost, which causes the node to go to the wait state often and induces a large delay. Indeed, the expected number of visits of the wait state is 0.32 when $\alpha=0.9$ and $\gamma=0,0.07$ when $\alpha=1$ and $\gamma=0$. As a result, the reduction of delay between $\alpha=0.9$ and $\alpha=1$ for $\gamma=0$

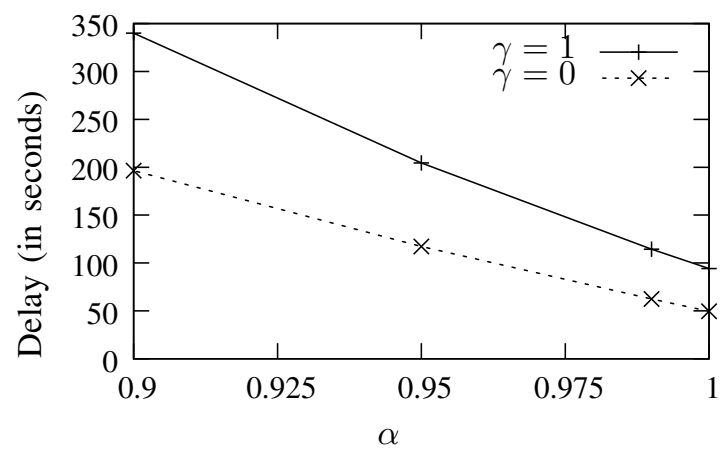

Figure 3. The expected duration of an activation is greatly impacted by the channel quality $\alpha$ and by $\gamma$.

is $146.9 \mathrm{~s}$ (as the wait state has a very large duration, which is $577 \mathrm{~s}$ in our setup $)^{7}$. The impact of $\gamma$, which determines the choice of receive window, is also significant for the delay. Indeed, when $\gamma=1$, collisions with transmissions from other nodes are likely to occur during either the transmission of the join request, or the transmission of the join accept. These collisions cause the node to go back to the wait state. When $\gamma=0$, collisions can occur during the transmission of the join request, but they cannot occur during the transmission of the join accept, as the gateway is the only node allowed to transmit on the channel dedicated to the second receive window. Thus, the number of expected visits to the wait state reduces, which in turn reduces the expected delay.

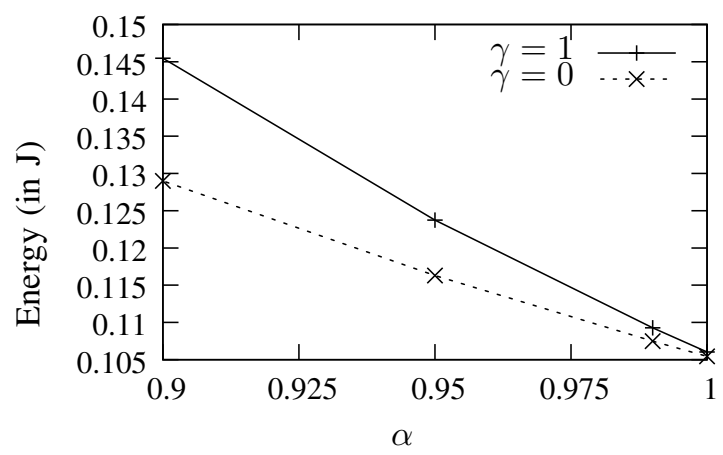

Figure 4. The expected energy consumption of an activation depends on the channel quality and $\gamma$ as well.

Figure 4 presents the energy as a function of $\alpha$ and $\gamma$. The impact of the channel quality $\alpha$ on the expected energy consumption is important. Indeed, a bad channel quality yields to frame loss and thus forces a node to go back to the wait state often in order to reach the activated state. The larger energy consumption when $\gamma=1$ is the energy cost of retransmitting join requests (and additional listening periods).

Figure 5 presents the delay as a function of the total number of channels and the number of available sub-bands $n_{S B}$. The $\mathrm{X}$-axis represents the total number of channels, which is the

\footnotetext{
${ }^{7}$ Note that the difference in the number of visits of the wait state accounts for about $145.1 \mathrm{~s}$ of these $146.9 \mathrm{~s}$. The extra delay is due to changes in the number of visits of the other, shorter states.
} 


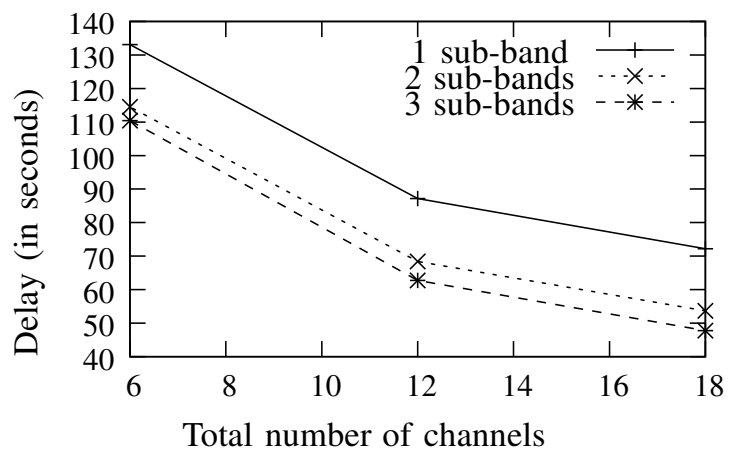

Figure 5. The expected duration of an activation depends on the total number of channels and the number of sub-bands.

product of $n_{C}$ and $n_{S B}$. The expected delay decreases with the number of channels as collisions during the transmission of the send request are less likely when there is a large number of channels. The expected delay is impacted by the number of sub-bands in two ways: (i) when the number of sub-bands increases, the time duration of the wait state is reduced as it is directly impacted by the number of sub-bands, and (ii) when the number of sub-bands increases, the traffic increases (as $\delta$ and $\tau_{A}$ are defined per sub-band), which increases the delay. However, the reduction of the wait duration is more significant than the increase in the number of collisions. Overall, the reduction in delay when $n_{S B}$ varies from one to three is between $19 \%$ (for a total number of channels of six) and $49 \%$ (for a total number of channels of eighteen).

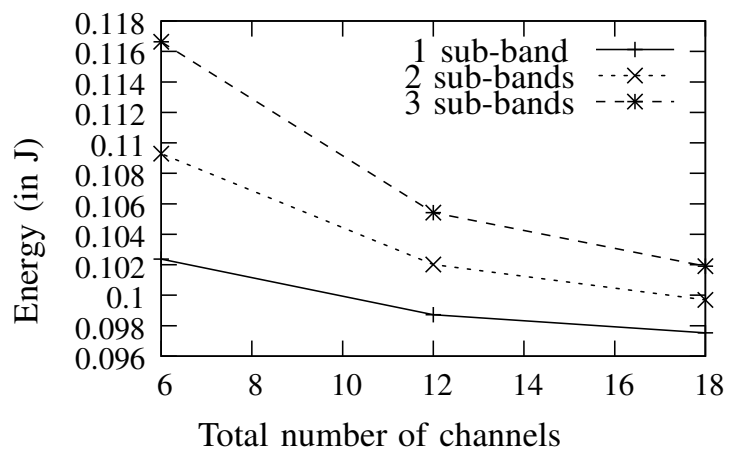

Figure 6. The expected energy consumption depends on the total number of channels, and on the number of available sub-bands.

Figure 6 presents the energy consumption of an activation as a function of the total number of channels and on the number of available sub-bands $n_{S B}$. The energy consumption decreases with the total number of channels as the probability of collisions decreases when the number of channels per subband is large. Moreover, when $n_{S B}$ is large, the node transmits join requests more frequently (as the duration of the wait state depends on the number of sub-bands). Overall, we notice an increase of $6 \%$ in energy consumption when two sub-bands are used and an increase of $13 \%$ when three sub-bands are used.

Figure 7 presents the delay as a function of the number of nodes and proportion of inactivated nodes, in three scenarios:

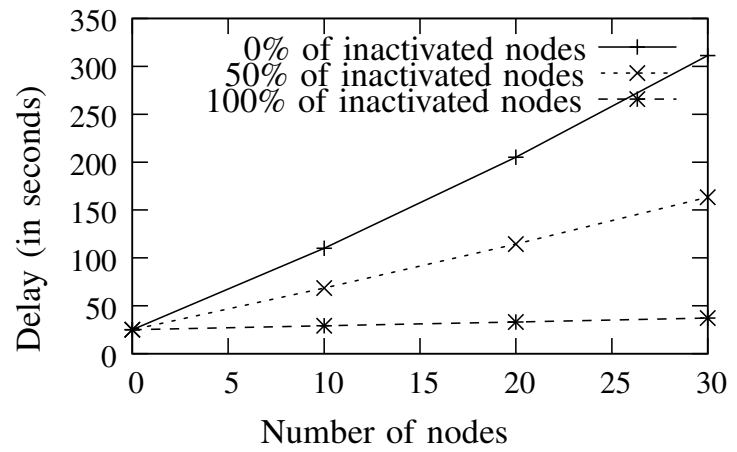

Figure 7. The expected delay is mainly impacted by the number of activated nodes.

when no node is activated, when half of the nodes are activated, and when all nodes are activated. The delay increases with the number of nodes in the network for all the scenarios. In the first scenario, the energy increases slightly as all nodes send their join requests with a small duty cycle of $0.1 \%$. Thus, collisions do not happen frequently and the number of visits of the wait state remains small. In the second scenario, the delay increases consistently. Indeed, the activated nodes send data frames with a larger duty cycle $\delta$ of $1 \%$. In this case, collisions and interferences appear more frequently and thus the number of visits of the wait state increases. The worst case scenario is the third scenario, in which all nodes are activated. All nodes send data frames with a large duty cycle $\delta$ of $1 \%$, and thus, the delay increases drastically.

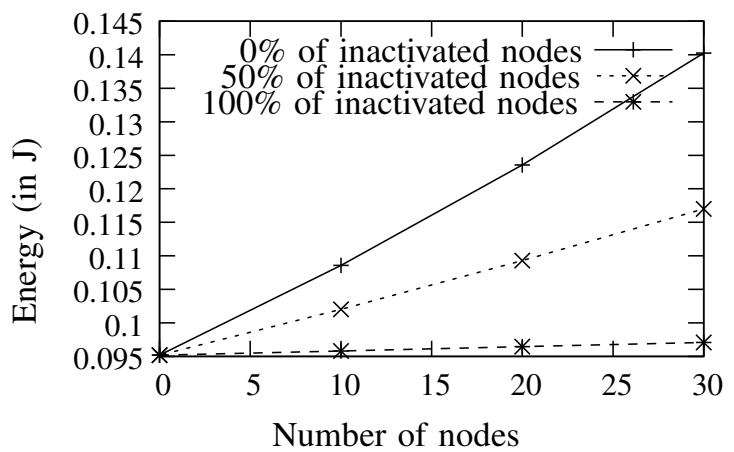

Figure 8. The expected energy consumption increases with the number of activated nodes.

Figure 8 presents the energy as a function of the number of nodes and proportion of inactivated nodes. The energy consumption follows the same behavior as the delay as it depends on the time spent (and the energy consumed) in each state. When the number of activated nodes increases in the network, the energy consumption of an activation increases as the number of visits to the send request state (which is the state consuming the most energy) increases. Moreover, the energy consumption increases with the number of nodes in the network as the number of collisions increases in this case. 


\section{DISCUSSION}

In this section, we discuss the impact of the regional settings on the model, as well as the impact of different data rates.

\section{A. Regional settings}

The Markov model described in this paper is based on the EU 863-870MHz ISM band (Section 7.1 of [14]). The standard describes three other regional settings.

The US 902-928 MHz ISM band (Section 7.2 of [14]) presents significant differences with the EU $863-870 \mathrm{MHz}$ ISM band. In the US band, there are $64+8=72$ default uplink channels and 8 default downlink channels (instead of 6 default channels used for both uplink and downlink, and one specific downlink channel). Because of the dedicated downlink channels, there is no interference between frames from the gateway and frames from other nodes. The probability of interference from other nodes is very small due to the large number of uplink channels. The probability of overhearing a frame from the gateway to another node is small too, due to the relatively large number of downlink channels. Join requests are sent alternatively on one of the 64 uplink channels with DR0, and on one of the 8 channels with a faster data-rate (namely, DR4 $)^{8}$. Finally, the data rates are larger than with the EU $863-870 \mathrm{MHz}$ setting (since DR0 uses a spreading factor of 10 instead of 12 , since DR4 is the minimum data rate for the 8 uplink channels, and since the data rate of downlink channels is much larger than DR0).

The China 779-787MHz ISM band (Section 7.3 of [14]) is similar to the EU $863-870 \mathrm{MHz}$ setting. The only difference is the maximum transmission power, which is smaller than with the EU 863-870MHz setting, and thus reduces the energy consumption (as well as the communication range).

The EU 432MHz ISM band (Section 7.4 of [14]) is similar to the EU $863-870 \mathrm{MHz}$ setting. The only difference is the number of default channels, which is only three (plus a dedicated channel for the second receive window).

\section{B. Impact of data rates}

The Markov model depends on the data rates used for join requests, join accepts and data frames. For instance, the probability $p_{c_{1}, r_{2}}$ increases with the data rate, as with larger data rates, larger frames can be received and discarded in less than $1 \mathrm{~s}$ (which is the time between the two receive windows). The probability of collisions also decreases with the data rate, as frames spend less time on the air. Also, the reduction of the time on air yields a reduction of the wait state duration, which has a significant impact on the overall delay.

\section{Conclusions}

LoRaWAN is a recent, promising technology that is expected to be used in several large-scale monitoring applications in both rural and urban environments. In this paper, we proposed to study its MAC layer performance. To do this, we proposed a Markov chain model for the on-the-air activation

\footnotetext{
${ }^{8}$ Modeling this alternation would require to split several states (including the send request state) in two in the Markov chain model.
}

procedure of the MAC layer. In this model, we took in account interferences with other nodes sending data frames or join request frames. We showed that the expected delay and energy consumption depend on network parameters such as number of channels per sub-band, number of sub-bands, and gateway configuration. We believe that this first study of the MAC layer of LoRaWAN will improve the understanding of the behavior and performance of this new standard.

Acknowledgment: This work has been partially funded by the Clervolc laboratory of excellence.

\section{REFERENCES}

[1] L. M. L. Oliveira, "Wireless Sensor Networks: a Survey on Environmental Monitoring," Journal of communications, vol. 6, no. 2, 2011.

[2] V. M. Rohokale, P. N. R., and R. Prasad, "A cooperative Internet of Things (IoT) for rural healthcare monitoring and control," in Wireless Communication, Vehicular Technology, Information Theory and Aeroscpace Electronic Systems Technology (Wireless ViTAE), 2001, 2nd Internal Conference on, 2011, pp. 1-6.

[3] X. Fafoutis, T. Sorensen, and J. Madsen, "Energy Harvesting - Wireless Sensor Networks for Indoors Applications Using IEEE 802.11," Procedia Computer Science, vol. 32, pp. 991-996, 2014.

[4] IEEE 802.15, "Part 15.4: low-rate wireless personal area networks (LRWPANs)," IEEE, Standard for local and metropolitan area networks IEEE Std 802.15.4-2011, 2011.

[5] _ "Part 15.4: wireless medium access control (MAC) and physical layer (PHY) specifications for low-rate wireless personal area networks (LR-WPANs)," ANSI/IEEE, Standard 802.15.4 R2003, 2003.

[6] A. Zanella, , N. Bui, A. Castellani, L. Vangelista, and M. Zorzi, "Internet of Things for Smart Cities," IEEE Internet of Things Journal, vol. 1, pp. 22-32, 2014.

[7] Z. Tao, S. Panwar, D. Gu, and J. Zhang, "Performance analysis and a proposed improvement for the IEEE 802.15.4 contention access period," in IEEE Wireless Communications and Networking Conference (WCNC), 2006.

[8] P. Park, P. Di Marco, P. Soldati, C. Fischione, and K. H. Johansson, "A generalized Markov chain model for effective analysis of slotted IEEE 802.15.4," in IEEE International Conference on Mobile Adhoc and Sensor Systems (MASS), 2009.

[9] E. D. Ngangue Ndih, N. Khaled, and G. De Micheli, "An analytical model for the contention access period of the slotted IEEE 802.15.4 with service differentiation," in IEEE International Conference on Communications (ICC), 2009.

[10] D. Striccoli, G. Boggia, and L. A. Grieco, "A Markov model for characterizing IEEE 802.15.4 MAC layer in noisy environments," IEEE Transactions on Industrial Electronics, 2014.

[11] Z. Xiao, C. He, and L. Jiang, "An analytical model for IEEE 802.15.4 with sleep mode based on time-varying queue," in IEEE International Conference on Communications (ICC), 2011.

[12] Sigfox, http//www.sigfox.com.

[13] Ingenu, http//www.ingenu.com.

[14] N. Sornin, M. Luis, T. Eirich, T. Kramp, and O. Hersent, "LoRaWAN Specification," LoRa Alliance, Standard V1.0, 2015.

[15] M. Centenaro, L. Vangelista, A. Zanella, and M. Zorzi, "Long-range communications in unlicensed bands: the rising stars in the IoT and smart city scenarios," 2015, under submission. arXiv:1510.00620 [cs.NI].

[16] Semtech, "LoRa ${ }^{\mathrm{TM}}$ modulation basics," Semtech Corporation, application note 1200.22, May 2015, revision 2.

[17] Libelium, "Waspmote LoRa $868 \mathrm{MHz} 915$ MHz SX1272 networking guide," Libelium Comunicaciones Distribuidas S. L., Tech. Rep., November 2015, version 4.2 . 